\title{
Multi-terminal HVDC grids with inertia mimicry capability
}

\author{
Weiyi Zhang ${ }^{1}$, Kumars Rouzbehi ${ }^{凶}$, Alvaro Luna ${ }^{1}$, Gevork B. Gharehpetian ${ }^{2}$, Pedro Rodriguez ${ }^{1,3}$ \\ ${ }^{1}$ Department of Electrical Engineering, Technical University of Catalonia, Rambla Sant Nebridi, Terrassa, Barcelona, Spain \\ ${ }^{2}$ Department of Electrical Engineering, Amirkabir University of Technology, Tehran, Iran \\ ${ }^{3}$ Abengoa Research, Abengoa, Palmas Altas, Sevilla, Spain \\ 凶E-mail: kumars.rouzbehi@upc.edu
}

\begin{abstract}
The high-voltage multi-terminal dc (MTDC) systems are foreseen to experience an important development in the next years. Currently, they have appeared to be a prevailing technical and economical solution for harvesting offshore wind energy. In this study, inertia mimicry capability is added to a voltage-source converter-HVDC grid-side station in an MTDC grid connected to a weak ac grid, which can have low inertia or even operate as an islanded grid. The presented inertia mimicry control is integrated in the generalised voltage droop strategy implemented at the primary level of a two-layer hierarchical control structure of the MTDC grid to provide higher flexibility, and thus controllability to the network. Besides, complete control framework from the operational point of view is developed to integrate the low-level control of the converter stations in the supervisory control centre of the MTDC grid. A scaled laboratory test results considering the international council on large electric systems (CIGRE) B4 MTDC grid demonstrate the good performance of the converter station when it is connected to a weak islanded ac grid.
\end{abstract}

\section{Introduction}

Lately, significant industrial and research studies in the topic of multi-terminal dc (MTDC) grid have been conducted all over the world [1]. From 1950 to 2015, around 180 two-terminal high voltage direct current (HVDC) transmission lines have been put into operation around the world. Over the past 20 years, some HVDC applications have been expanded with more than two terminals to obtain the first functional MTDC systems [2, 3]. With the development and increasing availability from multiple producers of high-power voltage-source converters (VSCs), the outlook of MTDC grids, composed of multiple VSC converter stations, has become a promising possibility [3]. Significant advantages and application ideas have been identified and proposed with regard to the MTDC grid concept in [4-10]. The MTDC grids could be one of the most suitable solutions for the integration of harvested wind farm energy into the mainland ac or island grids [10]. Furthermore, MTDC grids could facilitate the development of the so-called European supergrid [11-15], and this large-scale MTDC grid would finally interconnect the North Sea wind farms with the Mediterranean solar plants and Scandinavian hydropower. In addition, the MTDC applications can be found for the interconnection of multiple non-synchronous ac areas [14, 16, 17]. In general, MTDC grids have emerged as a promising solution to meet the requirements of the future electrical networks.

In normal operation of MTDC grids, the strength of the connected ac grids is an important factor. An ac grid can be considered as a weak grid from two point of views: first, high ac-grid impedance [18] and second, low ac-generation inertia. A typical high-impedance system can be found when a VSC-HVDC station is terminated to a weak point of a large ac grid, where the short-circuit capacity of the ac grid is low. On the other hand, low-inertia grids are considered to have a limited number of rotating machines or no rotating machine. Examples of such applications can be found when a VSC-HVDC station powers an island system such as offshore oil and gas platforms or a remote island that is powered through a VSC-HVDC link. It may also be the case when an HVDC link is initially connected to a large ac grid, but comes into island operation due to the trip of a critical ac transmission lines connected to the large ac grid or in case of black start after a global blackout of the grid. Synchronous condensers have been the traditional means to increase the inertia of an island system $[19,20]$. They also increase the short-circuit capacity of the ac grid. No matter if high impedance or low inertia is the issue considered, it can generally be solved by installing synchronous condensers. However, synchronous condensers increase considerably the investment and the maintenance costs of the project.

For interconnecting high-impedance ac grids, a grid synchronisation mechanism for VSC-HVDC that is alternative to the phase-locked loop (PLL) is proposed in [21] to emulate the synchronisation mechanism of synchronous machines. Nevertheless, the inertia emulation is not specifically discussed. Silva et al. [22] propose to use a time derivative of the frequency of the offshore grid to regulate wind power output, and further link the offshore frequency, dc voltage and onshore frequency by external droop controllers. In this manner, the offshore grids can contribute an amount of inertia to stabilise the frequency disturbances in the onshore grids. In [23, 24], inertia emulation control by VSC-HVDC is also developed, in which the dc voltage is regulated following the ac frequency in a specified trajectory based on the inertia characteristics, and the energy stored on the dc bus acts as the inertia reserve. Boundaries of the strategies in [22-24] can be the forming of an island grid and the inertia dynamics coupling by the dc voltage controller.

This paper investigates the potentials of the receiving-end VSC station of MTDC grid with capability of inertia mimicry when connected to an islanded ac grid. By using the proposed strategy, the total inertia of the island grid can be increased, thus contribute to the frequency stabilisation. Besides, since the VSC station mimics the behaviour of a synchronous machine, it is less limited by the short-circuit capacity of the ac grid. Such a control strategy is particularly suitable for island operation, where the ac-system conditions often vary to a large extent. The major focus of this paper is centred in the dynamic modelling and mainly in the control design. Moreover, a complete control framework for the operation of MTDC grid is also presented.

This paper is organised as follows. In Section 2, the hierarchical control for MTDC grids is introduced, followed by the description 
of the control strategy for VSC stations with generalised voltage droop (GVD) characteristics [25]. The complete control structure for the candidate VSC station with inertia mimicry capability is proposed in Section 3, where the GVD controller is also integrated for the operation modes smooth transition of the VSC stations. Scaled-down laboratory tests are conducted to validate the effectiveness of the proposed strategy.

\section{MTDC grid operation with GVD characteristics}

MTDC grids are characterised by the meshed interconnection of VSC-HVDC stations via cables, overhead lines or both. The main components of each VSC-HVDC station include: a VSC, a dc capacitor, an ac circuit breaker, an ac transformer, an ac filter and a phase reactor. In the following, the hierarchical control framework of the VSC stations and the primary control with GVD characteristics are briefly presented.

\subsection{Hierarchical control framework of MTDC grid}

For conducting an effective control and operation of an MTDC grid, a two-layer hierarchical control framework inspired by the ac grid control and operation approach is developed as shown in Fig. 1.

In this structure, the set points of the voltage droop controllers are generated at the secondary control layer, according to the grid data and the generation and demand requirements of the ac grid, and sent to the primary control layer. The secondary algorithm is based on the optimal power flow programme [26]. Then, the set point of active power or direct voltage and the reactive power or ac voltage set point for each converter station will be determined. Particularly, the set points of active power, direct voltage or droop slopes are translated to GVD controller parameters [25].

The primary control layer of MTDC grids includes the GVD controller, the reactive power controller, the ac voltage controller and the inner control loops. Commonly, the overall control objectives of an MTDC grid control consist of the dc voltage regulation at the dc terminals, control of active and reactive powers at the point of common coupling (PCC) and maintaining the PCC's ac voltage at the specified set point. On the basis of the provided secondary set points, the GVD controller is able to

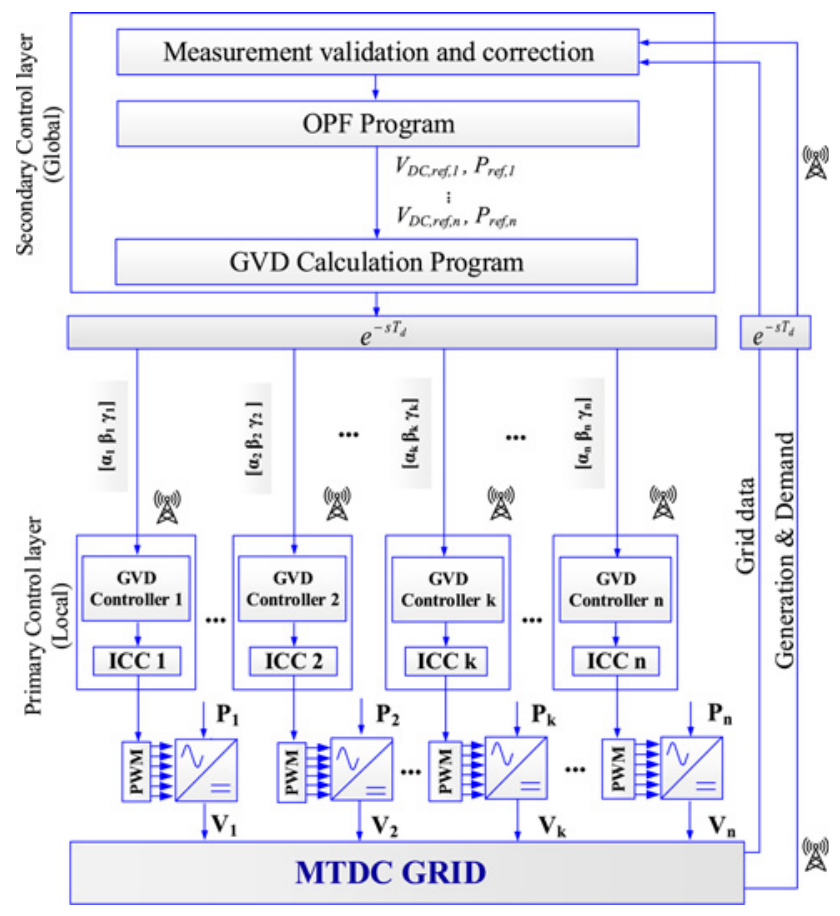

Fig. 1 Proposed hierarchical control framework for MTDC grids operate and switch in three different modes: active power control, dc voltage control or droop control. On the other hand, the reactive power controller or the ac voltage controller will be activated based on the roles dispatched to the converter station. The inner loops of the primary control layer are mainly responsible for the current regulation, for which the synchronous frame $(d q)$ current control paradigm is normally adopted.

\subsection{GVD characteristics}

The GVD control is proposed as a generalised form for integrating droop control, dc voltage control and active power control. Besides, the smooth mode transition capability brought by the GVD control creates more possibilities and flexibility in the commands and VSC stations management compared with the traditional voltage droop scheme that is only capable of power-voltage droop control.

The GVD characteristics are shown in Fig. 2. As illustrated in [25], the power control mode and dc voltage control mode can be, respectively, activated by designating $\alpha_{k}$ to 0 and $\beta_{k}$ to a small value, and the droop mode can be activated by specifying $\alpha_{k}$ and $\beta_{k}$ to result in the desired droop slope. In the meantime, the offset parameter $\gamma_{k}$ should be determined according to the optimal operation point found by the power flow programme.

On the basis of the GVD algorithm, a generalised reference for the followed proportional-integral (PI) controller will be generated. This reference can be the active power set point, the result of a droop-based power reference or the voltage reference. The active power control loop and dc voltage control loop have the same compensator form, i.e. the PI controller, which makes the GVD control feasible. Moreover, by scheduling the GVD parameters and the gains of the followed PI controller, smooth transition among different operation modes can be realised.

\section{Control of VSC station with inertia mimicry capability}

Harvesting energy from wind farms and delivering it to the mains and islands is one of the main attractive applications of MTDC grids. As a popular grid management approach, one station would be connected to a stiff ac grid performing voltage control acting as dc-slack bus, and stations connected to wind farms perform power control, while other stations perform power-voltage droop control.

In reality, the strength of the island grids that interface the MTDC grid is small, and hence it would be helpful to give inertia mimicry capability to the VSC station that interfaces the weak island grid. To achieve this important objective, in this paper an effective control strategy for the VSC station interfacing weak island grid is proposed.

\subsection{Overall control scheme}

The proposed control structure for VSC station capable of mimicking inertia is shown in Fig. 3.

The typical inner current controllers for VSC-HVDC stations are replaced by a structure featured by the inertia mimicry and virtual admittance block, which acts similar to the synchronisation mechanisms and output impedance of synchronous machines. The inputs of the controller as shown in Fig. 3 are dispatched by the secondary control layer commands.

The GVD strategy introduced in the former section is implemented to generate the reference for the inertia mimicry block. Since the VSC station(s) with inertia mimicry capability will feed an island grid and does not work as the main dc voltage governor, the GVD controller for this station will not act in fixed voltage control mode, but only in fixed power control mode or power-voltage droop mode. Besides, the proposed control can also be used for wind farm stations, while the inertia mimicry block set point needs to be specified by the output of a frequency controller instead of the GVD block in order to deliver the power following the wind state. 


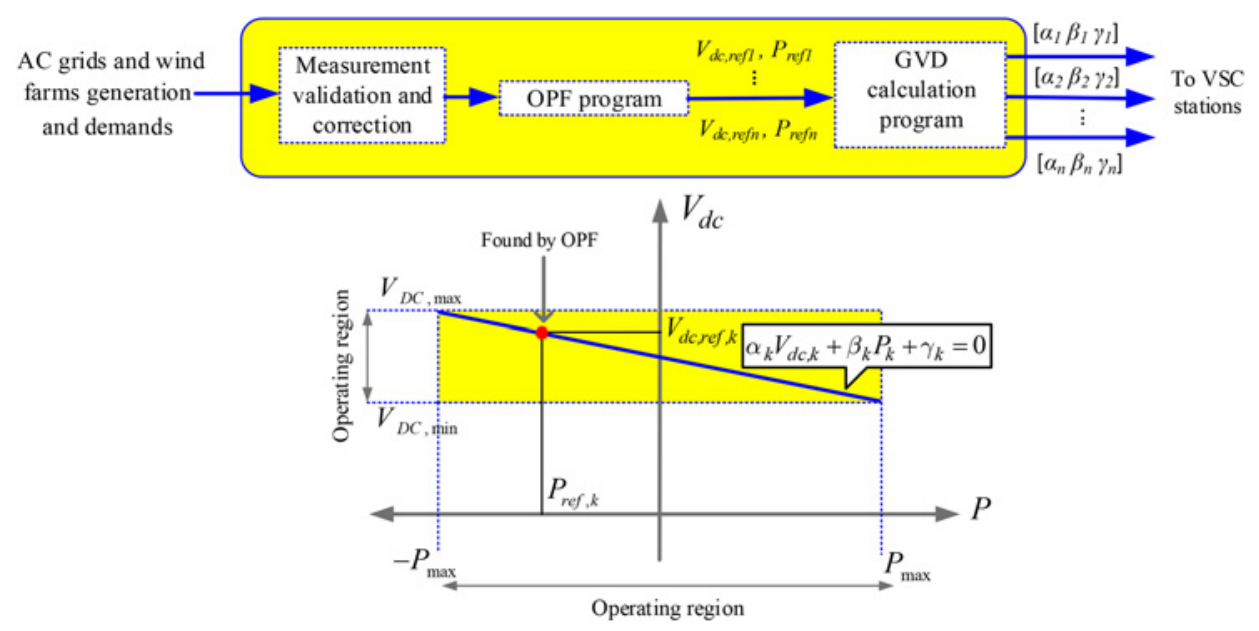

Fig. 2 GVD characteristics

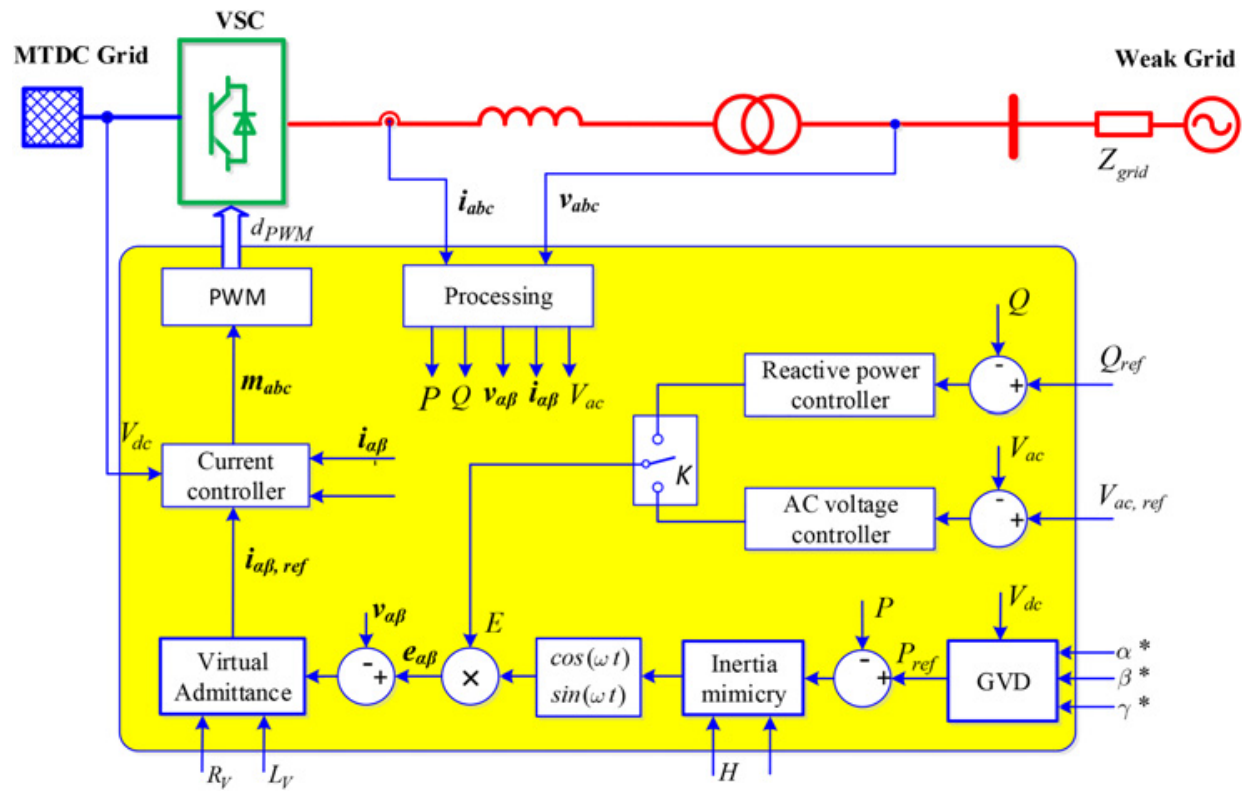

Fig. 3 Overall control scheme for VSC-HVDC station(s) with inertia mimicry capability

The output impedance of synchronous machines is emulated based on an admittance block and a current regulating loop, and the admittance block is expressed as

$$
\boldsymbol{i}_{\alpha \beta}^{r e f}=\frac{\boldsymbol{e}_{\alpha \beta}-\boldsymbol{v}_{\alpha \beta}}{R_{v}+s L_{v}}
$$

By setting different values to the virtual admittance, it is possible to present different responses to different frequency ranges and optimise the performance of the candidate VSC station according to the control requirements. The admittance block is more feasible for the realistic implementation compared with the impedance block due to the absence of derivative term that is contained in the impedance block [27] and the simplicity in configuring the converter to be a current source rather than a voltage source. The resistance and inductance of the virtual admittance can be either set according to the predefined values or secondary control layer commands.

The current control is achieved by a fast stationary frame current regulator which is expressed in (2), and the frequency signal generated by the outer loop is used as the concerned frequency of the applied resonant controller

$$
\boldsymbol{m}_{\alpha \beta}=\frac{1}{V_{d c}}\left(K_{P}+K_{R} \frac{\omega S}{S^{2}+\omega^{2}}\right)\left(i_{\alpha \beta}^{r e f}-i_{\alpha \beta}\right)
$$

where $m_{\alpha \beta}$ is the modulation reference on stationary frame, and $K_{\mathrm{P}}$ and $K_{\mathrm{R}}$ are, respectively, the proportional and resonant (PR) gains of the compensator.

The virtual synchronous frequency is generated by the inertia mimicry block, which is expressed as

$$
\omega=\omega_{\mathrm{ref}}+\left(K_{X}+\frac{K_{H}}{s}\right)\left(P_{\mathrm{ref}}-P\right)
$$

As shown in (3), the compensator form is a regular PI controller, and by designating the gains of the controller, the specified inertia and damping feature can be achieved. Same to the virtual admittance, the inertia and damping can be either predefined or adaptive according to the secondary control layer commands. The power reference $P_{\text {ref }}$ is generated by the GVD block. The design of the inertia mimicry block will be further illustrated in the next section. 
The magnitude of the virtual electromotive force can be generated by either the reactive power controller or the ac voltage controller, and the state of the switch $K$ should be determined according to the control requirement. The reactive power and ac voltage controller can be, respectively, realised by

$$
\begin{gathered}
E=E_{\mathrm{ref}}+\left(Q_{\mathrm{ref}}-Q\right)\left(K_{\mathrm{P} Q}+\frac{K_{\mathrm{I} Q}}{s}\right) \\
E=E_{\mathrm{ref}}+\left(V_{\mathrm{ac}, \mathrm{ref}}-V_{\mathrm{ac}}\right)\left(K_{\mathrm{P} A}+\frac{K_{\mathrm{I} A}}{s}\right)
\end{gathered}
$$

\subsection{Power control loop with inertia mimicry capability}

Fig. 4 shows the small-signal modelling of the power regulating loop, which is derived from the control scheme of Fig. 3. The designed power loop controller (PLC) and the virtual admittance correspond to the mechanical part and the electrical part of synchronous machines, respectively. The inertia mimicry characteristics can be specified by designing the PLC $G_{\text {PLC }}(s)$. For studying the dynamics, the grid frequency is also considered as a variable as well as the power reference, and it is linked to the grid angle by an integrator.

The power transfer of synchronous machines is related to the output reactance $X$ and is expressed as

$$
P=\frac{E V}{X} \sin \delta=P_{\max } \sin \delta
$$

where $E, V$ are the line-to-line root mean square (RMS) of the internal and output voltages, respectively, and $\delta$ is the load angle. Equation (6) can be expressed as (7) considering small amounts of $\delta$

$$
P=P_{\max } \delta
$$

Since the virtual admittance (accompanied by the current controller) is an emulation of the output impedance of synchronous machines, (7) is used for the modelling of the inner loops of VSC as seen in Fig. 4. When the PLC is designed to provide a certain amount of inertia, the time response of the current loop will be significantly faster than the power loop, and the dynamics of the current loop can hence be decoupled from the modelling of the power loop.

The PLC can be designed in different forms to achieve a zero steady-state error. One approach is to emulate the swing equation of synchronous machines for emulating the inertia characteristics [28], and then the resulting inertia mimicry block will be

$$
\omega=\omega_{\text {ref }}+\frac{1}{\omega_{\mathrm{s}}\left(J_{S}+D\right)}\left(P_{\text {ref }}-P\right)
$$

where $\omega_{\mathrm{s}}$ is the synchronous angular frequency, $J$ is the moment of inertia, and $D$ is the damping factor.

In this paper, the commonly used PI controller expressed in (3) is proposed as the compensator for the power loop for inertia mimicry. Compared with the design of (8), the PI controller is able to fix the steady-state power to the reference value even under grid frequency deviations, as well as presenting the inertia dynamics. In this way,

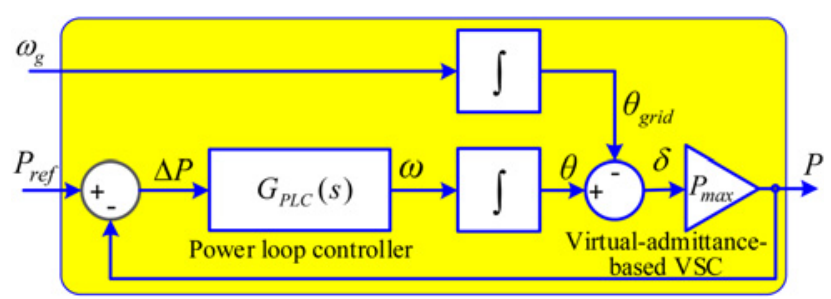

Fig. 4 Modelling of the power control loop with inertia mimicry the steady-state active power is completely controlled by the input of GVD controller, while the inertia effect dominates the dynamics.

\subsection{Power loop small-signal stability}

On the basis of the proposed inertia mimicry control, equivalence between VSC and synchronous machine is built. In traditional power systems, the small-signal rotor angle stability has to be achieved to maintain the synchronism of different machines. The instability can be the result of insufficient synchronising torque, insufficient damping torque or unstable control actions [29].

For synchronous machines, the issues of insufficient synchronising torque and unstable control actions are associated to the provision of primary source and the control of exciters and governors, respectively. Correspondingly for VSCs in MTDC systems, these two issues are associated to the available power from the dc side and the secondary control. Since the coordination of the generation and demand is not the focus of this paper, only the local stability of VSC is studied now, which corresponds to the stability issue of insufficient damping torque of synchronous machines.

The state-space representation of the power control loop in Fig. 4 is

$$
\begin{gathered}
\Delta \dot{\theta}=\boldsymbol{A} \Delta \theta+\boldsymbol{B}\left[\begin{array}{c}
\Delta P_{r e f} \\
\Delta \omega_{g}
\end{array}\right] \\
\Delta P=\boldsymbol{C} \Delta \theta+\boldsymbol{D}\left[\begin{array}{c}
\Delta P_{r e f} \\
\Delta \omega_{g}
\end{array}\right]
\end{gathered}
$$

where $\Delta \theta$ is seen as the state, and $\Delta P$ is the output, while $\Delta P_{\text {ref }}$ and $\Delta \omega_{\mathrm{g}}$ are the inputs. Moreover

$$
\begin{gathered}
\left.\boldsymbol{A}=[A]=-P_{\max } G_{P L C}(s)\right] \\
\boldsymbol{B}=\left[\begin{array}{ll}
B_{1} & B_{2}
\end{array}\right]=\left[\begin{array}{ll}
G_{P L C}(s) & \frac{1}{s} P_{\max } G_{P L C}(s)
\end{array}\right] \\
\boldsymbol{C}=[C]=\left[P_{\max }\right] \\
\boldsymbol{D}=\left[\begin{array}{ll}
D_{1} & D_{2}
\end{array}\right]=\left[\begin{array}{ll}
0 & -\frac{1}{s} P_{\max }
\end{array}\right]
\end{gathered}
$$

When (3) is specified as the power loop controller, the characteristic equation of the matrix $\boldsymbol{A}$ can be extracted as

$$
s^{2}+2 \xi \omega_{\mathrm{n}} s+\omega_{\mathrm{n}}^{2}=0
$$

where the damping factor $\xi$ and the natural frequency $\omega_{\mathrm{n}}$ are

$$
\begin{gathered}
\xi=\frac{K_{X}}{2} \sqrt{\frac{P_{\max }}{K_{H}}} \\
\omega_{\mathrm{n}}=\sqrt{P_{\max } K_{H}}
\end{gathered}
$$

By specifying the damping factor of the characteristic equation $\xi>0$, two eigenvalues of the matrix $\boldsymbol{A}$ will have negative real parts. Then, the control loop will be asymptotically stable according to Lyapunov's first method.

As seen in (12), the control parameters $K_{H}$ and $K_{X}$ can determine the inertia and damping characteristics, respectively.

To link the inertia characteristics to the inertia constant $H$ to be more straightforward in parameters setting, the controller form (8) is specified as the power loop controller in contrast. In this condition, the characteristic equation of the matrix $\boldsymbol{A}$ is the same as (11), whereas the damping factor $\xi$ and the natural frequency $\omega_{\mathrm{n}}$ are shown in (13). Thus, the parameter $K_{H}$ can be translated to the moment of inertia $J$ by combining (12b) and (13b), and can be 
further expressed in inertia constant $H$

$$
\begin{gathered}
\xi=\frac{D}{2} \sqrt{\frac{\omega_{\mathrm{s}}}{J P_{\max }}} \\
\omega_{\mathrm{n}}=\sqrt{\frac{P_{\max }}{J \omega_{\mathrm{s}}}}
\end{gathered}
$$

The response to the change of power reference $P_{\text {ref }}$ and grid frequency $\omega_{\mathrm{g}}$ can be estimated by the associated transfer functions. The relation between the power reference and the output power is

$$
\Delta P=\left[C(s-A)^{-1} B_{1}+D_{1}\right] \Delta P_{r e f}=\frac{\omega_{n}^{2}}{s^{2}+2 \xi \omega_{n} s+\omega_{n}^{2}} \Delta P_{r e f}
$$

Moreover, the relation between the output power and the grid frequency $\omega_{\mathrm{g}}$ can also be extracted as

$$
\Delta P=\left[C(s-A)^{-1} B_{2}+D_{2}\right] \Delta \omega_{g}=\frac{-P_{\max }\left(s+2 \xi \omega_{n}\right)}{s^{2}+2 \xi \omega_{n} s+\omega_{n}^{2}} \Delta \omega_{g}
$$

\subsection{Current loop stability}

The stability of the current regulating loop is a basic condition for the realisation of the virtual admittance, and it should be taken into account as an addition to the power loop stability. For specifying the gains of the PR current controller $K_{\mathrm{P}}$ and $K_{\mathrm{R}}$, the calculating algorithm based on phase margin and cross-over frequency can be used to guarantee a stability margin.

In discrete-time domain, the definitions of phase margin $\Phi m$ and cross-over frequency $\omega_{\mathrm{c}}$ are

$$
G_{O L}\left(e^{j \omega_{c} T_{s}}\right)=1 \angle-\left(180^{\circ}-\phi_{m}\right)
$$

where $G_{\mathrm{OL}}(\mathrm{z})$ is the discrete open-loop transfer function of the current loop and $T_{\mathrm{s}}$ is the sampling period.

The controller gains can be calculated once $\omega_{\mathrm{c}}$ and $\mathrm{phm}$ are fixed. In practice, the complex number (16) needs to be transformed into two real number equations by extracting the real and imaginary parts, respectively, as shown in (17)

$$
\begin{aligned}
& \operatorname{Re}\left(G_{P R}\left(e^{j \omega_{c} T_{s}}\right) \cdot G_{P}\left(e^{j \omega_{c} T_{s}}\right)\right)=\operatorname{Im}\left(1 \angle-\left(180^{\circ}-\phi_{m}\right)\right) \\
& \operatorname{Re}\left(G_{P R}\left(e^{j \omega_{c} T_{s}}\right) \cdot G_{P}\left(e^{j \omega_{c} T_{s}}\right)\right)=\operatorname{Im}\left(1 \angle-\left(180^{\circ}-\phi_{m}\right)\right)
\end{aligned}
$$

where $G_{\mathrm{PR}}(\mathrm{z})$ and $G_{\mathrm{P}}(\mathrm{z})$ are the transfer functions of the PR controller and the plant, respectively.

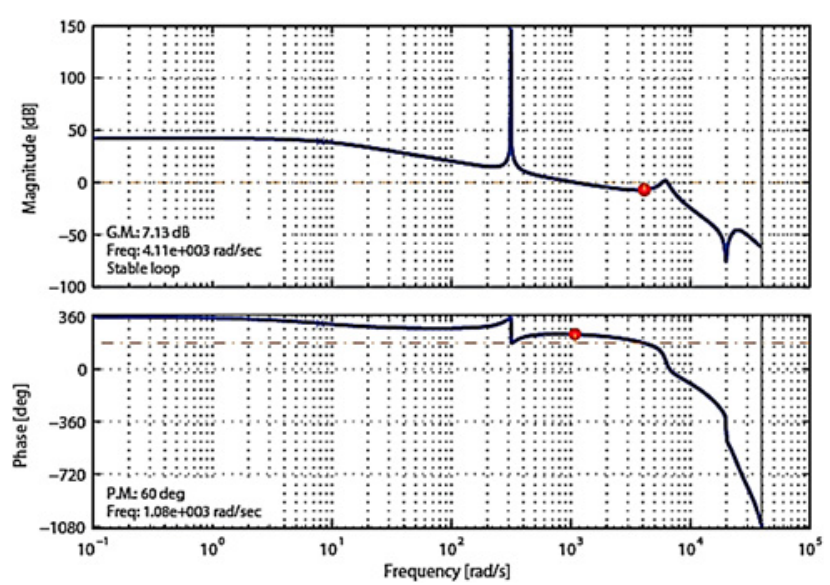

Fig. 5 Bode diagram of the current regulating loop under the selected controller gains
If $z_{\mathrm{c}}$ is defined to be the value of $z$ when $\omega$ is fixed to $\omega_{\mathrm{c}}$, then (17) is transformed into (18)

$$
\left[\begin{array}{ll}
K_{P} & K_{R}
\end{array}\right] \cdot\left[\begin{array}{cc}
1 & 0 \\
\operatorname{Re}\left(\operatorname{SOGI}\left(z_{c}\right)\right) & \operatorname{Im}\left(\operatorname{SOGI}\left(z_{c}\right)\right)
\end{array}\right]=\left[\begin{array}{ll}
\operatorname{Re}(a) & \operatorname{Im}(a)
\end{array}\right]
$$

In (18), the constant $a$ is expressed as

$$
a=\frac{1 \angle-\left(180^{\circ}-\phi_{m}\right)}{G_{P}\left(e^{j \omega_{c} T_{s}}\right)}
$$

Moreover, $\operatorname{SOGI}(\mathrm{z})$ is the discretised form of the resonant controller that is shown in (20) [30]

$$
\operatorname{SOGI}(z)=\frac{\omega_{0} T_{\mathrm{s}} z(z-1)}{(z-1)^{2}+\omega_{0}^{2} T_{\mathrm{s}}^{2} z}
$$

An optimal solution of the controller gains can be obtained by tuning $\omega_{\mathrm{c}}$ and the Bode plot of the open-loop system based on the selected controller gains is shown in Fig. 5, where the current loop stability is validated.

\subsection{Parameters setting for the inertia mimicry block}

The control parameters $K_{X}$ and $K_{H}$ can be clearly set according to the inputs of $\xi$ and $J$. Instead of using the moment of inertia $J$ to designate the inertia characteristics, the inertia constant $H$ is commonly adopted, which is defined in (21), meaning the time it takes to accelerate the rotational speed from 0 to $\omega_{\mathrm{s}}$ using full power $S_{N}$

$$
H=\frac{J \omega_{\mathrm{s}}^{2}}{2 S_{N}}
$$

Owing to the explicit relation between the controller gains and the characteristic parameters, the controller gains can be adaptive based on the inputs of $H$ and $\xi$, and a flexible control becomes possible.

$H$ reflects the short-term reserve from the dc side and can be seen equivalent to the inertia constant of synchronous machines. Moreover, the damping coefficient $\xi$ can be set considering the typical value range $0<\xi<1$ to create a stable and under-damped second-order system.

To predefine $\xi$, analysis on dynamics is done based on the mathematical transfer functions given in Section 3.3. A unitary

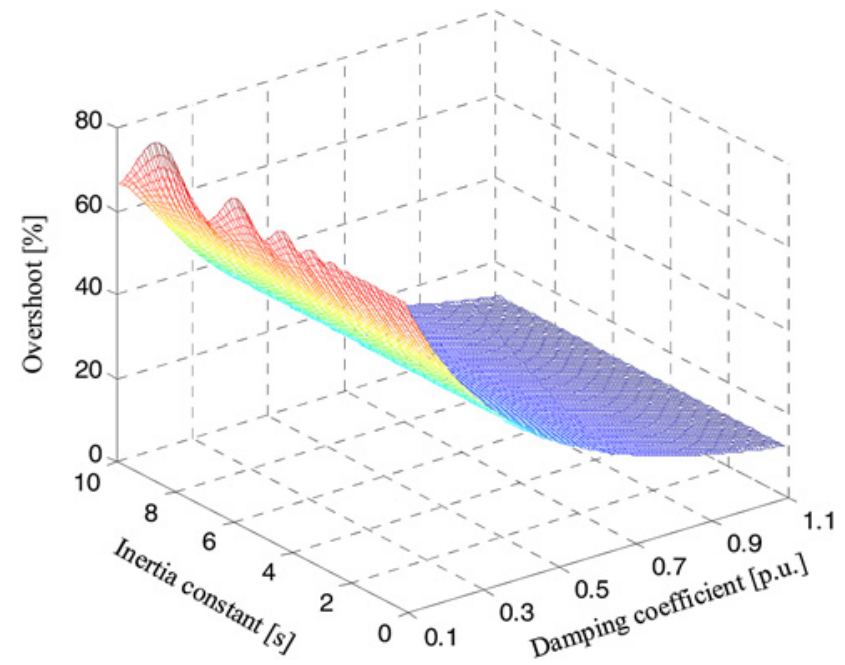

Fig. 6 Tuning of the damping coefficient 


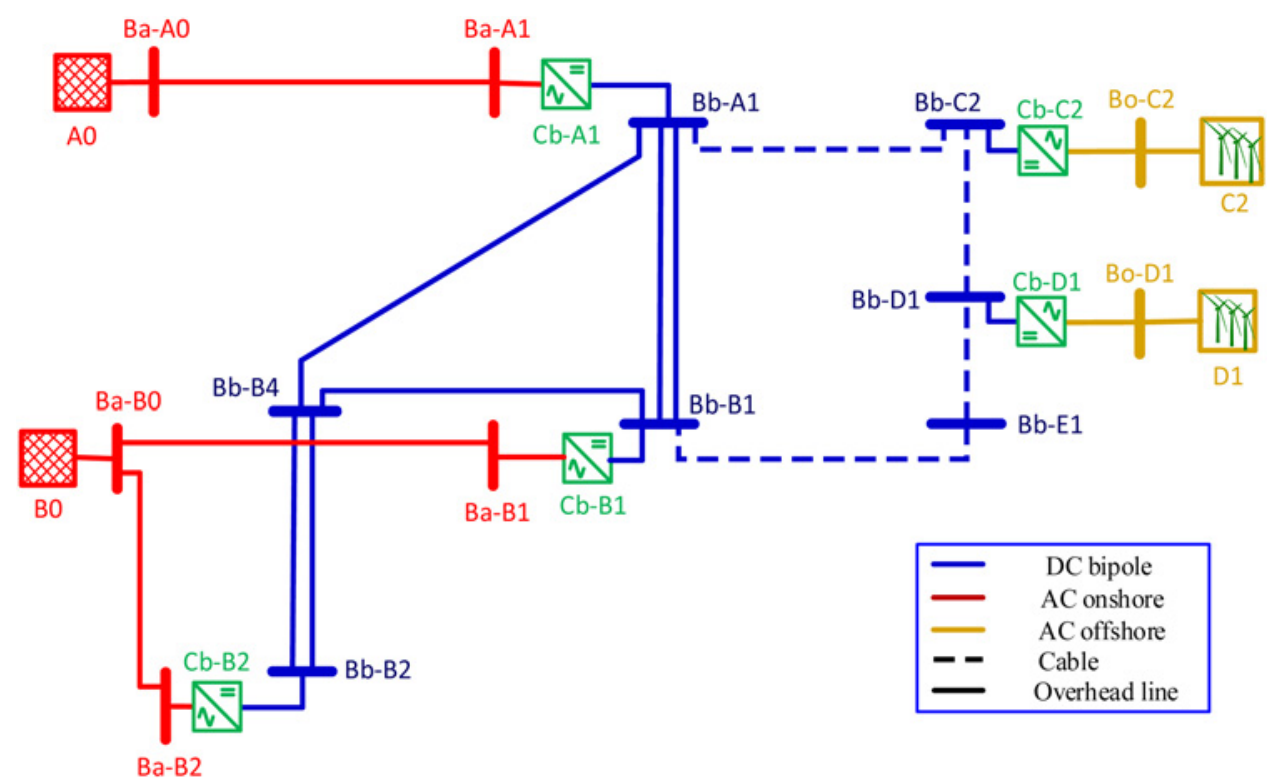

Fig. 7 CIGRE working group (WG) B4-58 MTDC test system

step input is given to the closed-loop transfer function (14), and the influences of $\xi$ and $H$ on the settling time and overshoot of the time response can be calculated. Then, $\xi$ can be adjusted to meet the requirements of the converter and grid. Analysis on the relation among $\xi, H$ and the overshoot of the power step response is shown in Fig. 6.

Since the overshoot of the step response can considerably indicate the damping performance of the system, a value bigger than 0.73 is proposed as the proper damping value, which can guarantee the

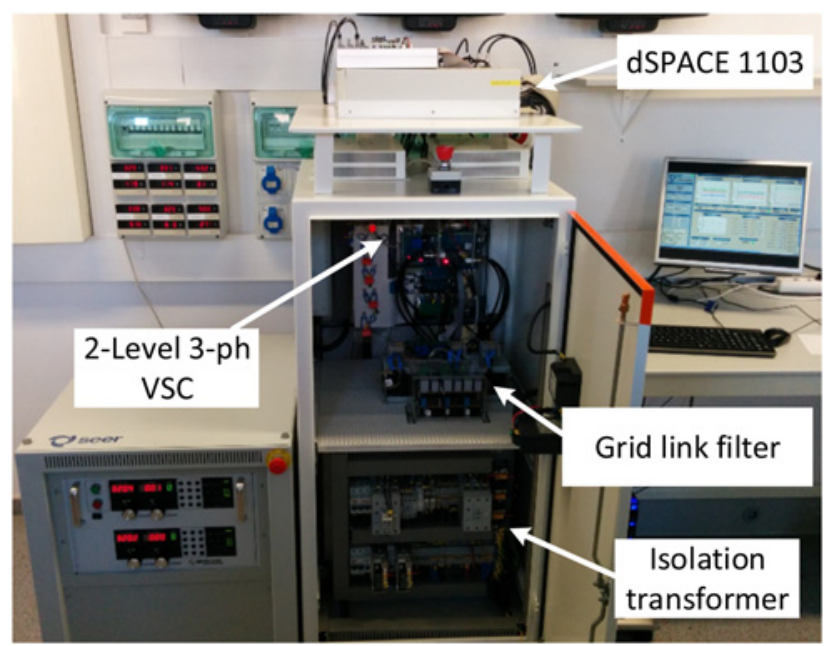

Fig. 8 Scaled laboratory prototype of the VSC Cb-Al as a candidate for inertia mimicry station

Table 1 Key parameters of the laboratory test system

\begin{tabular}{lcc}
\hline Symbol & Definition & Value \\
\hline$V_{\mathrm{DC}}$ & nominal dc-link voltage, $\mathrm{V}$ & 640 \\
$V_{\mathrm{g}}$ & nominal grid ph-to-ph voltage $\mathrm{RMS}, \mathrm{V}$ & 400 \\
$f_{\mathrm{g}}$ & grid nominal frequency, $\mathrm{Hz}$ & 50 \\
$S_{\mathrm{N}}$ & converter nominal power, $\mathrm{kW}$ & 10 \\
$f_{\mathrm{sw}}$ & switching frequency, $\mathrm{Hz}$ & 10,050 \\
$\xi$ & damping coefficient, pu & 0.73 \\
$R_{\mathrm{V}}$ & virtual resistance, pu & 0.1 \\
$X_{\mathrm{V}}$ & virtual reactance, pu & 0.3 \\
\hline
\end{tabular}

overshoot of the step response smaller than $20 \%$. It is worth mentioning that the inertia constant has very limited influence on the damping performance as shown in Fig. 6.

\section{Scaled-down laboratory results}

The CIGRE WG B4-58 MTDC test grid as shown in Fig. 7 is taken as an example of the application scenario of the proposed control strategy. The ac area A0 can be seen as an island grid that is connected to the MTDC grid through the converter station $\mathrm{Cb}-\mathrm{A} 1$, and then $\mathrm{Cb}-\mathrm{A} 1$ can be selected as the candidate VSC and controlled by the proposed control strategy to present inertia characteristics and support grid A0.

Table 2 Tests scenarios for the MTDC test grid under study

\begin{tabular}{lc}
\hline Test & Description \\
\hline 1 & the response of the converter influenced by the inertia \\
characteristics
\end{tabular}

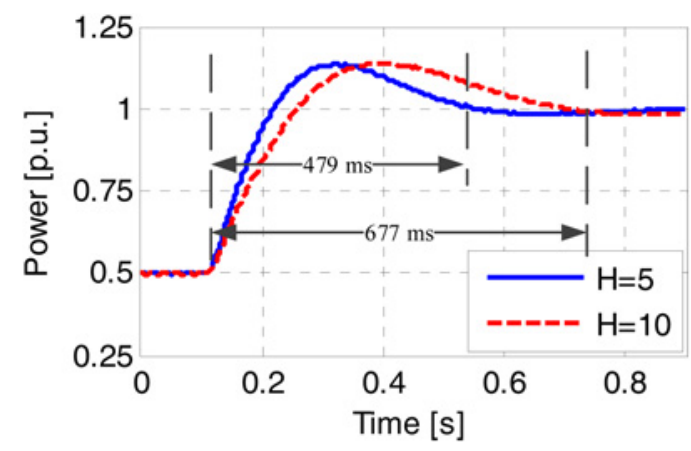

Fig. 9 Time responses in presence of a step change in power reference from 0.5 to $1 \mathrm{pu}$ 

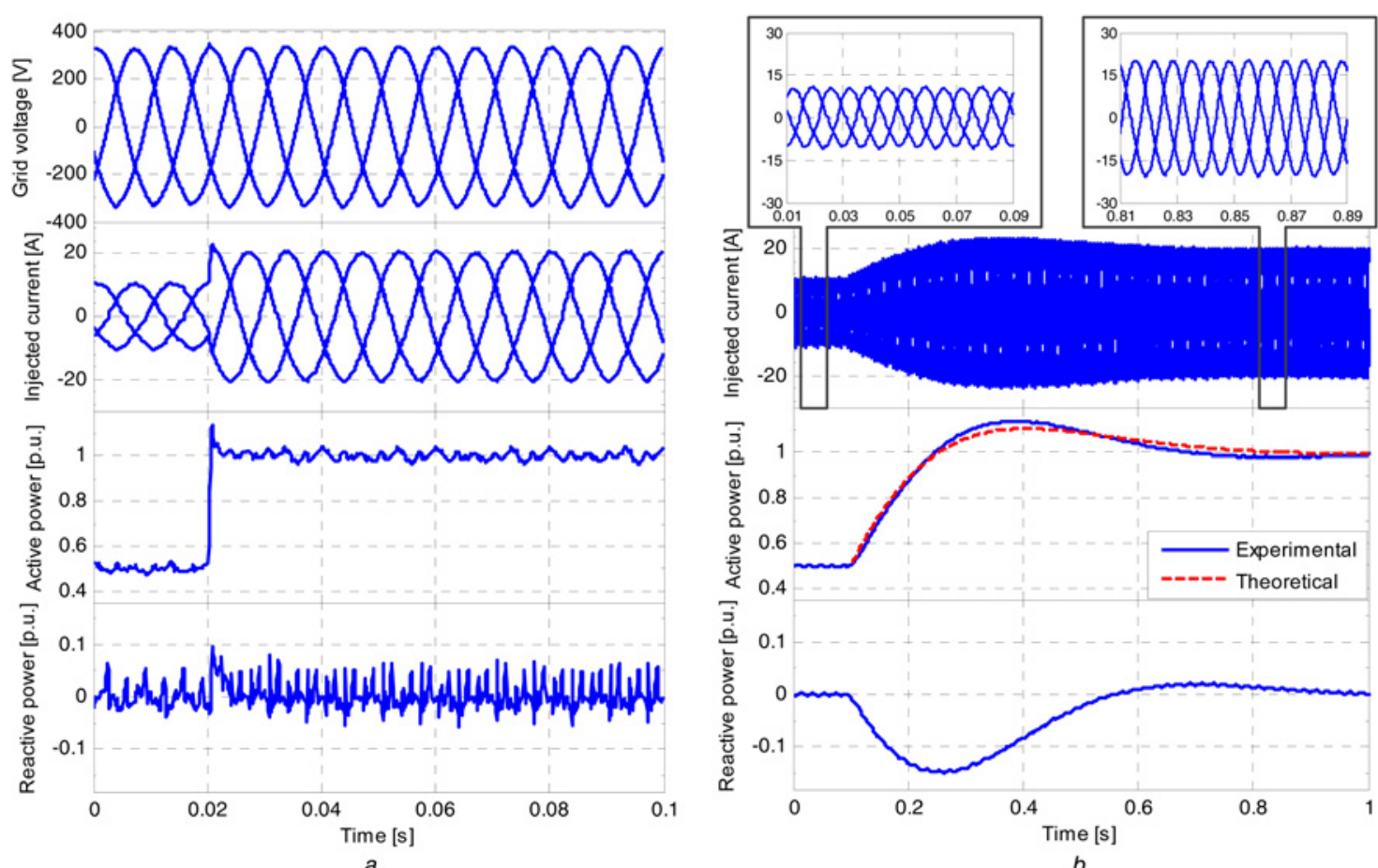

Fig. 10 Responses of the converter based on different strategies in presence of power reference step a Conventional strategy based on vector current control and PLL

$b$ Proposed inertia mimicry control

As an experimental validation, the converter station $\mathrm{Cb}-\mathrm{A} 1$ is scaled down to low-voltage laboratory prototypes as Fig. 8, and the proposed control strategy shown in Fig. 3 is implemented. The ac grid A0 is formed by a regenerative power source California Instruments MX45, by which the internal voltage profile, magnitude and frequency are programmable. By this instrument the sweep of grid frequency can be generated to emulate the frequency excursion in a low-inertia island grid. The output impedance of the regenerative source is specified to $0.002+$ $\mathrm{j} 0.002 \Omega$, thus the voltage profile at the PCC can be independent to the power injection from this VSC station. In this way, the power response of the VSC stations can hence be compared with the theoretical transfer functions (14) and (15) to validate the designed inertia characteristics. The key parameters of the setups and the controller are shown in Table 1.

For performing a clear evaluation and comparison of the experimental results, all the tests are conducted under the same specification of damping coefficient $\xi$, which is fixed to 0.73 . Moreover, the GVD controller works in power control mode. The results conducted in four test scenarios are summarised in Table 2.

Test case 1 studies the effect of the different values of the inertia constant. The time responses of the converter in presence of power reference step are shown, and the inertia constant $H$ is specified differently in two cases.

Fig. 9 shows the influence of the inertia constant $H$ on the time response of the power loop. The settling time $(2 \%$ steady-state band) when $H=10$ is close to the settling time when $H=5$ multiplied by $\sqrt{2}$. It matches the fact that the time response is proportional to the square root of $H$ approximately, since the natural frequency $\omega_{\mathrm{n}}$ is inversely proportional to the square root of $H$.

In test case 2, step changes in the power reference are given to validate the dynamic performance of the proposed control in contrast to the conventional inner and outer loop strategies for VSC-HVDC as shown in [25]. The inertia constant of the proposed strategy is specified to $10 \mathrm{~s}$. Both strategies achieve accurate control of active and reactive powers as shown in Fig. 10, while the inertia effect of the proposed control is explicitly exhibited by the significant difference in the dynamics of both strategies. Fig. $10 b$ also shows a comparison between the

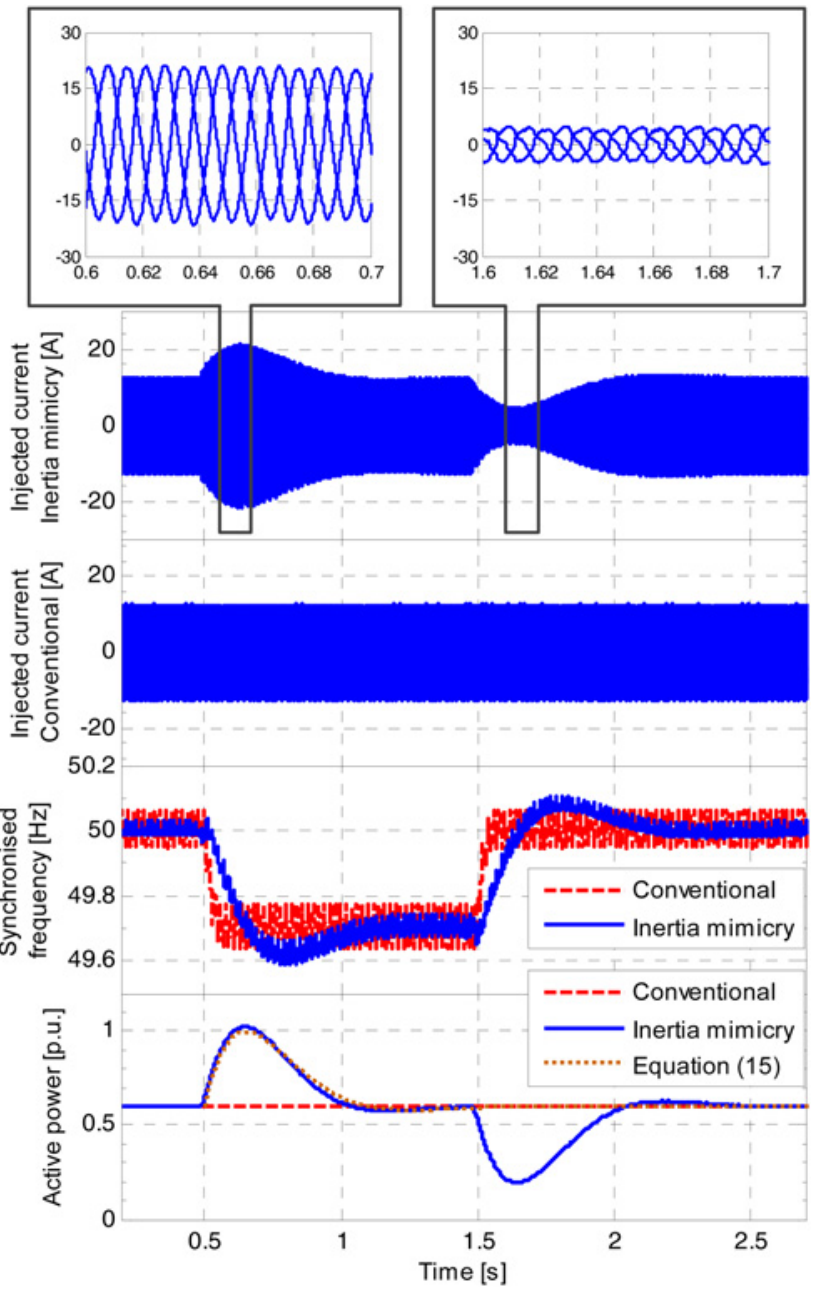

Fig. 11 Responses of the converter controlled by different strategies in presence of frequency sweep 

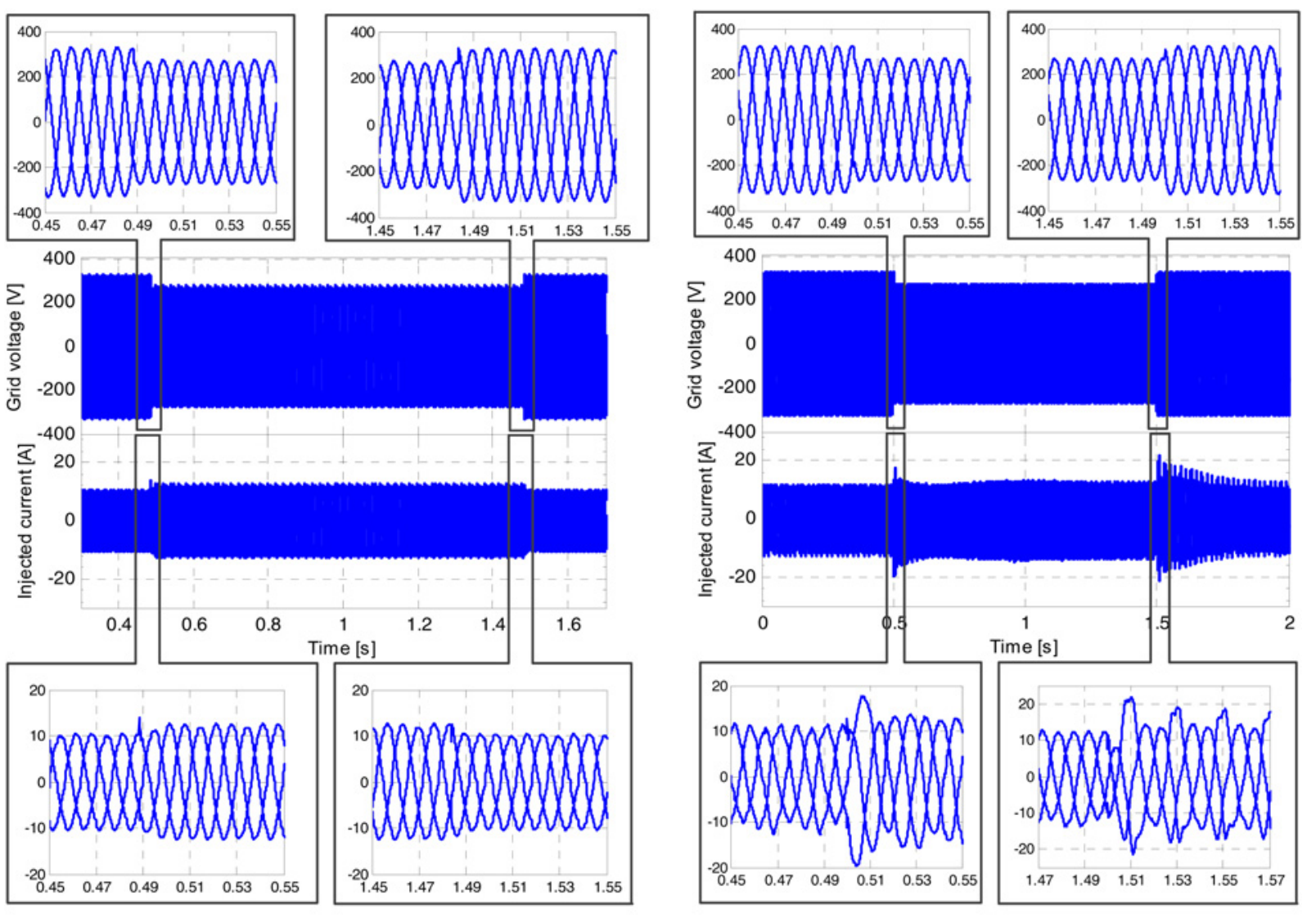

a

b

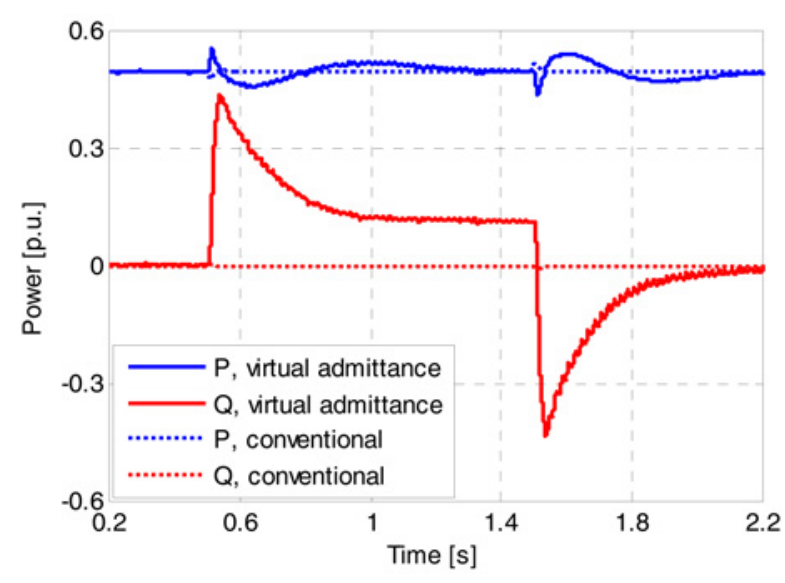

$c$

Fig. 12 Responses of the converter controlled by different strategies in presence of grid voltage dip

$a$ Conventional strategy based on vector current control and PLL

$b$ Proposed inertia mimicry control with virtual admittance

$c$ Comparison of reactive power injection of both strategies

theoretical response of active power calculated by the transfer function (14) and the profile of experimental data, which verifies the effective implementation of the designed inertia characteristics.

In test case 3, the frequency sweep of ac grid is given reflecting the disturbance of the island grid Ba-A0 due to the low-inertia generation. The frequency is designated to change at $0.5 \mathrm{~s}$ in a step from 50 to $49.7 \mathrm{~Hz}$ and maintains the state for $1 \mathrm{~s}$, and at $1.5 \mathrm{~s}$ change back to $50 \mathrm{~Hz}$ in a step. The steps in frequency are given only for evaluating the dynamics of the converter, even if the grid frequency will not experience sharp step changes in realistic applications.

The proposed strategy is compared with the conventional strategy as shown in Fig. 11. On the basis of the proposed strategy, the injected current and power will oppose the deviation of the grid frequency in transient, emulating the inertia characteristics of synchronous machines. In contrast, the conventional strategy has no inherent frequency response and simply maintains the synchronisation and power injection. Fig. 11 also shows both the synchronised frequency generated by the proposed inertia mimicry block and the classical stationary frame PLL [31]. Both signals show an accurate lock of the grid frequency in steady state; however, a more damped frequency measurement is seen by the proposed strategy, which can be a remarkable feature to contribute to weak ac grids connection and adverse grid conditions.

Besides, Fig. 11 also validates that the inertia dynamics comply with the transfer function (15). A step in frequency of $-0.3 \mathrm{~Hz}$ at $0.5 \mathrm{~s}$ is given as the input of (15), and the calculated response of power is plotted for comparison. An ideal match between the 
theoretical and experimental responses is found. Thanks to the inertia dynamics of the power loop, the reference of the current loop does not experience drastic changes, and hence the dynamics of the current loop and the non-linearity of the plant is not coupled in the response.

In test case 4, a voltage dip is given, and the results based on both the conventional and the proposed strategies are shown in Fig. 12.

The three-phase dip is generated and lasts for $1 \mathrm{~s}$, and the phase-to-neutral voltage RMS is decreased from 230 to $190 \mathrm{~V}$. During the dip, the converter under both control strategies keeps connected to the grid and the injected current is seen stable without significant oscillations. Fig. $12 c$ shows that the proposed control gives a significant voltage support behaviour by injecting reactive power during the voltage dip owing to the virtual impedance.

\section{Conclusion}

In this paper, an inertia mimicry control for receiving-end stations in MTDC systems was developed for its connection to island systems or contributing inertia in the main grids. The load angle synchronisation mechanisms of synchronous machines were emulated in the designed control, and the PLL can hence be eliminated oriented to the constraints in interfacing high-impedance grids. The generation or loads perturbations in the ac grid would be damped by exchanging energy with the dc grid based on the inertia mimicry. In addition, the adjustable virtual admittance structure contained in the designed control emulates the output impedance of the synchronous machines, which enable the grid forming, natural load sharing and start-up without PLL. The scaled-down laboratory tests exhibited the expected performance defined by the proposed control and can be a preliminary validation of practicality of the hierarchical control framework.

\section{Acknowledgments}

This work has been partially supported by the Spanish Ministry of Science and Innovation under the project ENE2014-60228-R. Any opinions, findings and conclusions or recommendations expressed in this material are those of the authors and do not necessarily reflect those of the host institutions or funders.

\section{References}

1 Saeedifard, M., Graovac, M., Dias, R.F., et al.: 'DC power systems: challenges and opportunities'. IEEE PES General Meeting, 2010, pp. 1-7

2 'Atlantic wind connection', 2013. Available at http://www.atlanticwindconnection.com

3 'ABB HVDC systems', 2013. Available at http://www.new.abb.com/systems/hvdc

4 Barker, C.D., Whitehouse, R.: 'Autonomous converter control in a multi-terminal HVDC system'. Ninth IET Int. Conf. on ACDC, 2010, pp. 1-5

5 Jovcic, D., Lin, W.: 'Power balancing and dc fault ride through in DC grids with dc hubs and wind farms', IET Renew. Power Gener., 2015, 9, (7), pp. 847-856

6 Wai, R.-J., Liao, Y., Wang, G.-D.: 'Design of backstepping power control for grid-side converter of voltage source converter-based high-voltage dc wind power generation system', IET Renew. Power Gener., 2013, 7, (2), pp. 118-133

7 Xu, L., Yao, L.: 'DC voltage control and power dispatch of a multi-terminal HVDC system for integrating large offshore wind farms', IET Renew. Power Gener., 2011, 5, (3), p. 223
8 Eghtedarpour, N., Farjah, E.: 'Distributed charge/discharge control of energy storages in a renewable-energy-based DC micro-grid', IET Renew. Power Gener., 2014, 8, (1), pp. 45-57

9 Kumar, M., Srivastava, S.C., Singh, S.N., et al.: 'Development of a control strategy for interconnection of islanded direct current microgrids', IET Renew. Power Gener., 2015, 9, (3), pp. 284-296

10 Gomis-Bellmunt, O., Liang, J., Ekanayake, J., et al.: 'Topologies of multiterminal HVDC-VSC transmission for large offshore wind farms', Electr. Power Syst. Res., 2011, 81, (2), pp. 271-281

11 Van Hertem, D., Ghandhari, M.: 'Multi-terminal VSC HVDC for the European supergrid: obstacles', Renew. Sustain. Energy Rev., 2010, 14, (9), pp. 3156-3163

12 Xu, L., Williams, B.W., Yao, L.: 'Multi-terminal DC transmission systems for connecting large offshore wind farms'. IEEE PES General Meeting, 2008, pp. 1-7

13 Dierckxsens, C., Srivastava, K., Reza, M., et al.: 'A distributed DC voltage control method for VSC MTDC systems', Electr. Power Syst. Res., 2012, 82, (1), pp. $54-58$

14 da Silva, R., Teodorescu, R., Rodriguez, P.: 'Multilink DC transmission system for supergrid future concepts and wind power integration'. IET Conf. on Renewable Power Generation, 2011, pp. 1-6

15 Vrana, T.K., Torres-Olguin, R.E., Liu, B., et al.: 'The North Sea super grid - a technical perspective'. Ninth IET Int. Conf. on AC and DC Power Transmission, 2010, pp. $1-5$

16 Pinto, R.T., Bauer, P., Rodrigues, S.F., et al.: 'A novel distributed direct-voltage control strategy for grid integration of offshore wind energy systems through MTDC network', IEEE Trans. Ind. Electron., 2013, 60, (6), pp. 2429-2441

17 Dai, J., Phulpin, Y., Sarlette, A., et al.: 'Coordinated primary frequency control among non-synchronous systems connected by a multi-terminal high-voltage direct current grid', IET Gener. Transm. Distrib., 2012, 6, (2), p. 99

18 Martinez, A.: 'Connecting wind power plants to weak grids', 2015

19 Al-Haddad, K., Saha, R., Chandra, A., et al.: 'Static synchronous compensators (STATCOM): a review', IET Power Electron., 2009, 2, (4), pp. 297-324

20 Mendis, N., Muttaqi, K.M., Perera, S.: 'Management of battery-supercapacitor hybrid energy storage and synchronous condenser for isolated operation of PMSG based variable-speed wind turbine generating systems', IEEE Trans. Smart Grid, 2014, 5, (2), pp. 944-953

21 Zhang, L., Harnefors, L., Nee, H.P.: 'Interconnection of two very weak AC systems by VSC-HVDC links using power-synchronization control', IEEE Trans. Power Syst., 2011, 26, (1), pp. 344-355

22 Silva, B., Moreira, C.L., Seca, L., et al.: 'Provision of inertial and primary frequency control services using offshore multiterminal HVDC networks', IEEE Trans. Sustain. Energy, 2012, 3, (4), pp. 800-808

23 Zhu, J., Booth, C.D., Adam, G.P., et al.: 'Inertia emulation control strategy for VSC-HVDC transmission systems', IEEE Trans. Power Syst., 2013, 28, (2), pp. $1277-1287$

24 Zhu, J., Guerrero, J.M., Hung, W., et al.: 'Generic inertia emulation controller for multi-terminal voltage-source-converter high voltage direct current systems', IET Renew. Power Gener., 2014, 8, (7), pp. 740-748

25 Rouzbehi, K., Miranian, A., Luna, A., et al.: 'A generalized voltage droop strategy for control of multi-terminal DC grids', IEEE Trans. Ind. Appl., 2013, 51, (1), pp. 59-64

26 Rouzbehi, K., Miranian, A., Luna, A., et al.: 'DC voltage control and power sharing in multiterminal DC grids based on optimal DC power flow and voltage-droop strategy', IEEE J. Emerg. Sel. Top. Power Electron., 2014, 2, (4), pp. 1171-1180

27 Rodriguez, P., Candela, I., Citro, C., et al.: 'Control of grid-connected power converters based on a virtual admittance control loop'. Proc. EPE, 2013

28 Rodriguez, P., Candela, I., Rocabert, J., et al.: 'United states patent application publication: synchronous power controller for a generating system based on static power converters', 2014

29 Kundur, P.: 'Power system stability and control' (McGraw-Hill, New York, USA, 1994)

30 Rodríguez, F.J., Bueno, E., Aredes, M., et al.: 'Discrete-time implementation of second order generalized integrators for grid converters'. Proc. - 34th Annual Conf. of the IEEE Industrial Electronics Society, IECON 2008, 2008, no. 1, pp. 176-181

31 Rodríguez, P., Luna, A., Candela, I., et al.: 'Multiresonant frequency-locked loop for grid synchronization of power converters under distorted grid conditions', IEEE Trans. Ind. Electron., 2011, 58, (1), pp. 127-138 